\title{
Caracterización integral de la ataxia espinocerebelosa 2 en Cuba y su aplicación en proyectos de intervención
}

\section{Comprehensive characterization of spinocerebellar ataxia type 2 in Cuba and its application in intervention projects}

\begin{abstract}
Luis Velázquez Pérez,' Roberto Rodríguez Labrada," Gilberto Sánchez Cruz,"' J osé M. Laffita Mesa,'v Luis Almaguer Mederos, ${ }^{\mathrm{V}}$ Raúl Aguilera Rodríguez, vı Jacqueline Medrano Montero, VII Dennis Almaguer Gotay, VIII Tania Cruz Mariño, ${ }^{1 \mathrm{X}}$ Yanetza González Zaldívar, ${ }^{\mathrm{X}}$ Dany Coello Almarales, ${ }^{\mathrm{I}}$ Nalia Canales Ochoa, ${ }^{\prime \prime}$ Yaimeé Vázquez Mojena, ${ }^{x \prime \prime I}$ J ulio Cesar Rodríguez Díaz ${ }^{\text {IIV }}$
\end{abstract}

' Doctor en Medicina. Especialista de II Grado en Neurofisiología Clínica. Investigador Titular. Doctor en Ciencias Médicas. Centro para la Investigación y Rehabilitación de las Ataxias Hereditarias. Holguín, Cuba.

"Licenciado en Microbiología. Centro para la Investigación y Rehabilitación de las Ataxias Hereditarias. Holguín, Cuba.

III Doctor en Medicina. Especialista de II Grado en Medicina Interna. Centro para la Investigación y Rehabilitación de las Ataxias Hereditarias. Holguín, Cuba.

iv Licenciado en Microbiología. Centro para la Investigación y Rehabilitación de las Ataxias Hereditarias. Holguín, Cuba.

$\checkmark$ Licenciado en Biología. Doctor en Ciencias Médicas. Centro para la Investigación y Rehabilitación de las Ataxias Hereditarias. Holguín, Cuba.

VI Doctor en Medicina. Especialista de I Grado en Medicina Interna. Máster en Urgencias Médicas. Centro para la Investigación y Rehabilitación de las Ataxias Hereditarias. Holguín, Cuba.

VII Doctor en Estomatología. Especialista de I Grado en Ortodoncia. Centro para la Investigación y Rehabilitación de las Ataxias Hereditarias. Holguín, Cuba.

VIII Licenciado en Química. Máster en Biotecnología. Centro para la Investigación y Rehabilitación de las Ataxias Hereditarias. Holguín, Cuba.

IX Doctor en Medicina. Especialista de I Grado en Genética Médica. Centro para la Investigación y Rehabilitación de las Ataxias Hereditarias. Holguín, Cuba.

x Licenciada en Microbiología. Centro para la Investigación y Rehabilitación de las Ataxias Hereditarias. Holguín, Cuba.

${ }^{x I}$ Licenciado en Biología. Máster en Neurociencias. Centro para la Investigación y Rehabilitación de las Ataxias Hereditarias. Holguín, Cuba.

XII Licenciada en Enfermería. Centro para la Investigación y Rehabilitación de las Ataxias Hereditarias. Holguín, Cuba.

XIII Licenciada en Microbiología. Máster en Virología. Centro para la Investigación y Rehabilitación de las Ataxias Hereditarias. Holguín, Cuba.

XIV Licenciado en Cultura Física. Centro para la Investigación y Rehabilitación de las Ataxias Hereditarias. Holguín, Cuba. 


\section{RESUMEN}

Introducción Cuba es el país con mayores tasas de prevalencia e incidencia para las ataxias hereditarias, lo que constituye un problema de salud que motivó la creación del Centro para la Investigación y Rehabilitación de Ataxias Hereditarias en Holguín. Objetivos Describir los principales resultados, aportes científicos, estrategias de intervención e impactos que durante más de 10 años se han obtenido por el citado centro, como modelo para el abordaje integral de las ataxias hereditarias en Cuba. Fuente de datos Se realizó una revisión en las bases de datos Pubmed-Medline y Scopus, analizando todos los artículos relevantes, comprendidos en el periodo 19782011. Se utilizó el descriptor «ataxia espinocerebelar», de elevada especificidad y sensibilidad para el tema en análisis.

Síntesis de los datos La prevalencia de la enfermedad se ha mantenido constante durante 40 años, extendiéndose a toda la isla. La mutación ataxia espinocerebelosa tipo 2 es responsable del $60 \%$ de la variabilidad fenotípica mientras que el $40 \%$ restante se debe a factores modificadores genéticos y/o ambientales. Se ha descrito la existencia de un daño oxidativo severo, disminución de neuroprotectores y oligoelementos. Los estudios neurofisiológicos permitieron definir etapas evolutivas desde estadios preclínicos de la enfermedad así como biomarcadores de progresión y daño genético. Estos resultados propiciaron el diseño y ejecución de varios ensayos clínicos controlados en busca de un protocolo de tratamiento contra la enfermedad. Adicionalmente se brinda un servicio de diagnostico prenatal y presintomático con un impacto positivo sobre las familias afectadas.

Conclusiones Las investigaciones sobre la ataxia espinocerebelosa tipo 2 cubana, como problema de salud, han tenido un enfoque integral. Los nuevos descubrimientos sobre la patogenia, la identificación de biomarcadores, los ensayos clínicos, el diagnóstico prenatal y presintomático permitieron conformar un nuevo modelo cubano para el abordaje de las ataxias hereditarias y el estudio de otras enfermedades neurodegenerativas.

Palabras clave: Ataxia espinocerebelosa tipo 2, SCA2, ataxia cubana, Centro para la Investigación y Rehabilitación de las ataxias hereditarias (CIRAH).

\section{ABSTRACT}

Introduction Cuba is one of the countries with high rates of prevalence and incidence of hereditary ataxias, which is a health problem that encouraged the foundation of the Center for Research and Rehabilitation of Hereditary Ataxias in Holguín province.

Objectives To describe the main results, scientific achievements, intervention strategies and impacts of this institution for more than 10 years, as a sort of pattern to be followed to approach hereditary ataxias in Cuba in a more comprehensive way. Data source Pubmed-Medline and Scopus database were reviewed in which all the relevant articles published from 1978 to 2011 were analyzed. Spinocerebelar ataxia, highly specific and sensitive subject headings, were used for the topic under analysis. Data synthesis The prevalence of this disease has remained unchanged for 40 years, being extended to the whole island. Spinocerebelar ataxia type 2 mutation accounts 
for $60 \%$ of the phenotypical variability whereas the remaining $40 \%$ is caused by genetic and/or environmental modifying factors. Severe oxidative damage, reduction of neuroprotectors and of oligoelements have been described. The neurophysiological studies allowed defining evolutionary phases from the preclinical stagings as well as progression and genetic damage biomarkers. These results allowed designing several controlled clinical assays in search of one treatment protocol for the disease.

Additionally, prenatal and pre-symptomatic diagnosis service is rendered, with positive impact on affected families.

Conclusions The research studies on spinocerebelar ataxia type 2 in Cuba as a health problem have had comprehensive approach. The new breakthroughs on pathogeny, identification of biomarkers, clinical assays, prenatal and presymptomatic diagnosis allowed making a new Cuban model to approach hereditary ataxias and the study of other neurodegenerative diseases.

Key words: Spinocerebelar ataxia type 2, SCA2, Cuban ataxia, Center for Research and Rehabilitation of Hereditary Ataxias (CIRAH).

\section{NTRODUCCI ÓN}

El término ataxia, no define a una enfermedad específica ni a un diagnóstico determinado, sino al síntoma resultante del estado patológico de la coordinación de los movimientos. Con frecuencia, esta palabra se emplea para describir los trastornos de la marcha, los que se caracterizan por inestabilidad, incoordinación y aumento de la base de sustentación. ${ }^{1}$ Aunque las causas que originan el fenotipo atáxico pueden ser variadas, se conoce que este resulta de una disfunción a nivel del cerebelo y/o sus vías aferentes y eferentes, así como alteraciones en la médula espinal, nervios periféricos o una combinación de estas tres condiciones. ${ }^{1}$

De todas las formas de ataxias, las más comunes y por tanto las más estudiadas son las ataxias hereditarias. En la actualidad, con el vertiginoso desarrollo de la genómica y la proteómica se ha alcanzado un avance muy significativo en el conocimiento científico sobre estas enfermedades. Se han identificado cerca de 10 genes diferentes relacionados con ataxias hereditarias autosómicas recesivas, entre las cuales la ataxia de Friedreich presenta las mayores tasas de prevalencia e incidencia. ${ }^{2}$

Por su parte, las ataxias hereditarias autosómicas dominantes son comúnmente conocidas como ataxias espinocerebelosas (SCA, del inglés spinocerebellar ataxias). Estas comprenden un extenso grupo de enfermedades neurodegenerativas, caracterizadas por una gran heterogeneidad desde el punto de vista clínico, patológico y molecular. ${ }^{3}$ Actualmente se conocen 31 formas moleculares de SCAs, aunque la heterogeneidad genética observada entre estas sugiere que por lo menos faltan por identificar el $30 \%$ de la etiología molecular de las mismas. Hasta este momento se han identificado y caracterizado las mutaciones asociadas a 17 subtipos de SCAs. ${ }^{4}$

Una de las formas de ataxias autosómicas dominantes más comunes a nivel internacional es la ataxia espinocerebelosa tipo 2 (SCA2). ${ }^{1}$ Esta enfermedad alcanza las mayores tasas de prevalencia e incidencia en Cuba, donde las primeras observaciones epidemiológicas fueron realizadas entre las décadas del 60 y el 70 del pasado siglo, identificándose desde entonces una prevalencia significativamente 
elevada en la región oriental del país, con predominio en la región correspondiente a la actual provincia Holguín. ${ }^{5}$

En el año 1990, investigadores cubanos informan la más profunda caracterización clínica de la enfermedad, ${ }^{6}$ y a partir del año 1998 da inicio una nueva etapa en el proceso investigativo y asistencial de las ataxias hereditarias en Cuba caracterizada por una mayor organización de la actividad investigativa y se dieron los primeros pasos para la instauración de programas asistenciales. Esta etapa se consolida a partir del año 2000 con la creación del Centro para la Investigación y Rehabilitación de las Ataxias Hereditarias ( $\mathrm{Cl} R A H)$ en Holguín.

Esta entidad de Ciencia e Innovación Tecnológica del MINSAP consta de áreas especializadas en ciencias clínicas, biología molecular, genética predictiva, neurofisiología clínica y otras, con los recursos humanos y tecnológicos aptos para el desarrollo de investigaciones científicas y actividades asistenciales para las familias afectadas. Entre las principales contribuciones realizadas por esta institución aparecen: la instauración de un Programa de Neurorrehabilitación Multifactorial, la caracterización neurofisiológica de los sistemas somáticos y autonómicos, la identificación de factores modificadores de la edad de inicio y el curso evolutivo de la enfermedad, la instauración de un Programa de Diagnóstico Prenatal y Presintomático para la SCA2 y el desarrollo de un modelo animal transgénico para esta enfermedad, así como la implementación de los ensayos preclínicos y clínicos.

El objetivo de este trabajo es describir los principales resultados, aportes científicos, estrategias de intervención e impactos, que durante más de 10 años se han obtenido en el CIRAH, como modelo para el abordaje integral de las ataxias hereditarias en Cuba.

\section{FUENTE DE DATOS}

Se realizó una revisión bibliográfica en las bases de datos Pubmed, Medline y Scopus, en la que se analizaron todos los artículos relevantes sin restricción de modalidad de artículo o idioma. Los descriptores utilizados fueron: spinocerebellar ataxia type 2 , spinocerebellar ataxia 2, SCA2, Type 2 spinocerebellar ataxia, cuban ataxia, ataxia espinocerebelosa tipo 2, ataxia espinocerebelosa 2 y ataxia cubana. Para restringir la búsqueda a las contribuciones realizadas por el CIRAH, en el campo de las ataxias espinocerebelosas se incluyeron los siguientes descriptores en el campo de afiliación de los autores: Center for the Investigation and Rehabilitation of Hereditary Ataxias, Holguín, Cuba; Clinic for Research and Rehabilitation of Hereditary Ataxias, Holguín, Cuba; Clínica para la Investigación y Rehabilitación de las Ataxias Hereditarias (CIRAH), Holguín, Cuba; Clínica para Investigación y Rehabilitación de las Ataxias Hereditarias (CIRAH), Holguín, Cuba; Clínica Cubana para la Investigación y Rehabilitación de las Ataxias Hereditarias (CIRAH), Holguín, Cuba; Centro de Investigaciones y Asistencia Médica para la Ataxia Cubana, Holguín, Cuba. El periodo comprendido en la revisión fue desde 1978 (primeros informes del estudio de las ataxias hereditarias en Cuba) hasta el 2 de abril de 2011.

\section{SÍ NTESI S DE LOS DATOS}

\section{Epidemiología de la sca2}

La incidencia y prevalencia globales de la SCA2 son desconocidas debido a que la mayoría de los estudios epidemiológicos han sido desarrollados en regiones geográficas aisladas con familias no suficientemente numerosas como para realizar análisis de ligamiento y la organización de los sistemas de salud de la mayoría de los países carecen de las potencialidades objetivas para realizar este tipo de pesquisas.

http://scielo.sld.cu 
Sin embargo, el desarrollo de estudios de prevalencia en Cuba, se encuentran con una situación muy favorable debido a la existencia de un sólido sistema de salud pública aparejado a la creación de una red nacional de ataxia, el trabajo cooperado de varias instituciones y la contribución de todas las familias afectadas.

Hasta la fecha, se han desarrollo 3 estudios neuroepidemiológicos para las ataxias hereditarias en Cuba. El primero, realizado en la década del 70 en la región oriental, identificó una alta prevalencia de las ataxias autosómicas dominantes en la región geográfica del oriente-norte, con mayor significación en la actual provincia Holguín. ${ }^{5}$

En el año 1998, se realizó el segundo estudio, esta vez dentro de la provincia Holguín, donde se identifican a los municipios de Báguanos, Cacocum y Holguín, como lo más afectados. ${ }^{7}$ Finalmente, entre los años 2002 y 2003 bajo la conducción del CIRAH, se realizó un estudio neuroepidemiológico nacional que abarcó a la totalidad de los enfermos y descendientes en riesgo del país. ${ }^{8}$ Este último estudio permitió identificar 162 familias afectadas con algún tipo de ataxia autosómica dominante, las que agrupan a más de 660 enfermos y 7160 descendientes en riesgo en Cuba.

Para el caso específico del subtipo SCA2 se registran 101 familias, 562 enfermos y 7 068 descendientes, lo que indica una frecuencia superior al $80 \%$ entre el resto de las ataxias dominantes. Se notificaron pacientes en 12 de las 15 provincias, pero se identificaron descendientes en riesgo en todas. Esto representa una tasa estimada nacional de la mutación SCA2 de 20 casos por 100 mil habitantes en Cuba, con variaciones entre las diferentes provincias (Fig.1).

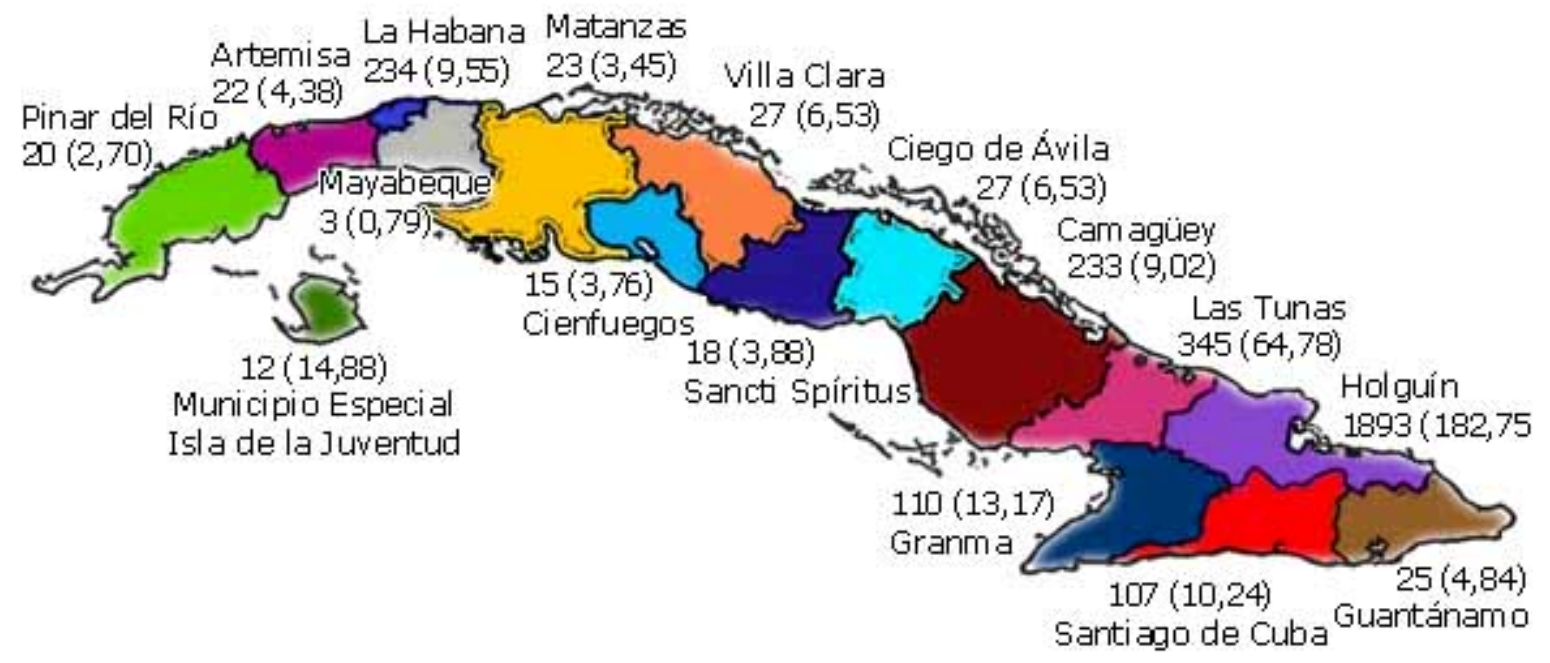

Fig. 1. Tasa de prevalencia de la mutación SCA2 en todas las provincias cubanas. Los valores fuera del paréntesis representan el número de pacientes y descendientes en riesgo y entre paréntesis, la tasa de prevalencia de la mutación.

En la provincia de Holguín se observa la mayor tasa de prevalencia del país ( 42 enfermos por cada 100 mil habitantes) con valores significativos en el municipio de Báguanos, donde los valores superan los 141 pacientes por 100 mil habitantes. ${ }^{8}$

\section{Caracterización clínica de la sca2}

Entre los principales aportes de Cuba al conocimiento de la ataxia SCA2 está la caracterización fenotípica de la enfermedad, lo que permite conocer la prevalencia de 


\section{Revista Cubana de Salud Pública. 2011;37(3):230-244}

cada síntoma y signo clínico. Lo anterior adquiere un valor muy importante desde el punto de vista diagnóstico. La caracterización clínica realizada por el grupo cubano también permite describir otros síntomas no muy comunes, que en poblaciones pequeñas de enfermos no podrían ser encontrados con significación estadística.

De esta manera se pudo determinar que las manifestaciones clínicas más frecuentes de la ataxia cubana son los trastornos de la marcha, los que se presentan en la totalidad de los enfermos. Otros síntomas muy frecuentes son la disartria cerebelosa y la dismetría, signos que prevalecen en más del $98 \%$ de los enfermos. La adiadococinesia o incapacidad para ejecutar movimientos alternativos rápidos se observa en cerca del $97 \%$ de los pacientes. ${ }^{1,8}$

Otras manifestaciones de menor prevalencia en la SCA2 cubana son la hipotonía (62 $\%)$, temblor cinético de miembros superiores e inferiores (58 \%), contracturas musculares dolorosas ( $57 \%$ ), signo de Romberg $(41 \%)$, arreflexia osteotendinosa de miembros inferiores (41\%) apalestesia en los miembros inferiores (30\%), hiporreflexia osteotendinosa ( $11 \%)$, clonus ( $10 \%)$, hiperreflexia osteotendinosa, signo de Babinski y trastornos de la sensibilidad superficial ( $5 \%$ ). ${ }^{1,8}$ Por otro lado, investigaciones cubanas han demostrado la existencia de síntomas y signos disautonómicos en varios pacientes. ${ }^{9,10}$

Otros signos de la enfermedad son los trastornos cognitivos, en especial las disfunciones frontoejecutivas, de la atención, concentración y la memoria. Sin embargo, es poco usual que los pacientes progresen hasta demencias. ${ }^{11}$

La edad de inicio de la SCA2 oscila entre 4 y 73 años, pero el valor medio ronda los 31 años de edad. La duración promedio de la enfermedad es de 15 años, aunque esta puede variar entre 6 y 50 años. Generalmente los pacientes debutan con la ataxia de la marcha ( $94 \%$ ), mientras que el $6 \%$ de ellos comienzan mostrando dificultades para hablar e incoordinación de los movimientos alternativos de los miembros superiores. ${ }^{1,8}$

\section{Biología molecular de la sca2}

Las investigaciones realizadas en Cuba y específicamente en el CIRAH han sido de gran importancia para el estudio de las características genéticas y moleculares de la SCA2. En el año 1993, investigadores cubanos y alemanes realizaron estudios de ligamientos en familias cubanas e identificaron al segmento cromosómico 12q23-24.1 como el sitio más probable donde aparece en gen de la enfermedad. ${ }^{12}$ En el año 1995, estos mismos investigadores identificaron un haplotipo común en 11 familias cubanas con ataxias, dando luz a las primeras evidencias sobre el efecto fundador de la enfermedad en Cuba. ${ }^{13}$ Un año más tarde, el grupo cubano en colaboración con la Universidad Central de Los Ángeles (UCLA) identifican el gen relacionado con la SCA2 y caracterizan la mutación causante de la enfermedad, la que consiste en una mutación dinámica descrita por la expansión del número de repeticiones del trinucleótido CAG en el primer exón del gen SCA2 por encima de 32 unidades. ${ }^{14}$ Esta alteración genética se traduce en la expresión de un dominio poliglutamínico expandido que le confiere propiedades tóxicas a la proteína mutada (ataxina 2), la que forma inclusiones proteicas citoplasmáticas. ${ }^{15}$

Estudios siguientes han demostrado que el principal efecto patogénico de la ataxina 2 mutada se expresa en las neuronas de Purkinje, localizadas en la corteza cerebelosa. En estas, la proteína se distribuye por toda la célula, incluyendo a las dendritas. Diversos estudios han revelado su presencia en la fracción citosólica y la fracción retículo endoplasmático/complejo de Golgi, así como a lo largo de la membrana citoplasmática y los polirribosomas. ${ }^{15,16}$ 
Otras de las principales contribuciones del CIRAH en el orden molecular ha sido la caracterización de la correlación genotipo-fenotipo en la SCA2. En tal sentido se ha demostrado que en la población cubana el tamaño de expansión del trinucleótido CAG influye en el $60 \%$ sobre la edad de inicio de la enfermedad en los pacientes con SCA $2,{ }^{17}$ y se han investigado los factores genéticos y no genéticos que influyen sobre el $40 \%$ restante de la variabilidad de la edad de inicio (Fig. 2). Estos estudios realizados en colaboración con las Universidades de Frankfurt, Alemania y la UCLA han identificado al tamaño del dominio poliglutamínico en el alelo no expandido de la ataxina 2 y del gen cacna I,${ }^{17}$ así como a la variante polimórfica del complejo mitocondrial $1,{ }^{18}$ como los principales factores genéticos modificadores de la edad de inicio.

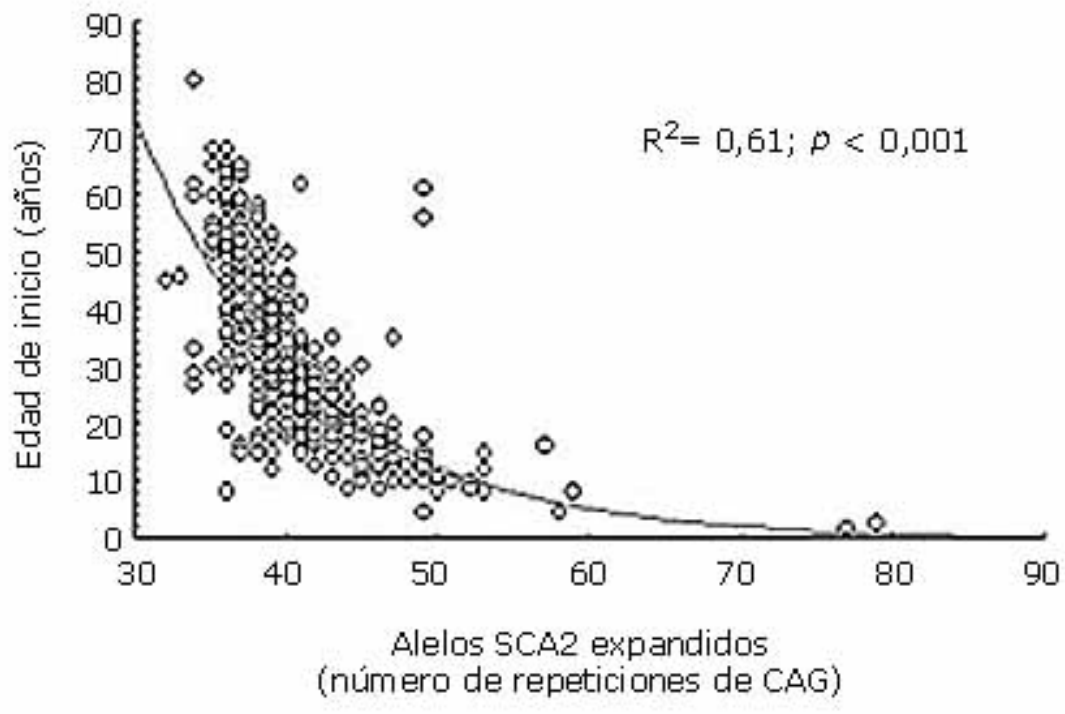

Fig.2. Influencia del número de repeticiones de CAG sobre la edad de inicio de la SCA2.

Otro aporte significativo del CIRAH es la generación del primer ratón transgénico para la SCA2 en el que el transgén está bajo el promotor fisiológico del gen, implicando que su expresión sea ubicua y que posea una cinética similar a la observada en humanos. ${ }^{19}$ Este modelo representa una valiosa herramienta para avanzar en el conocimiento de la patogénesis de la SCA2 y ha permitido realizar estudios en diferentes estadios del proceso patológico que por las características de la enfermedad son inaccesibles en humanos, así como probar potenciales tratamientos y evaluar sus efectos en ensayos preclínicos.

\section{Caracterización bioquímica de la SCA2}

El estudio de la SCA2 desde el punto de vista bioquímico representa una vía muy importante para identificar blancos terapéuticos y diseñar las correspondientes terapias. El CIRAH, en estrecha relación con diversas instituciones del polo científico de La Habana, como son el Centro para la Producción de Animales de Laboratorio, Centro de Investigación y Desarrollo de Medicamentos (CIDEM), Centro de Química Farmacéutica y el Instituto de Farmacia Alimentos, ha logrado caracterizar a la enfermedad desde el punto de vista bioquímico y enfatizado en los sistemas de defensa antioxidante y el estudio de oligoelementos.

El análisis de los niveles de estrés oxidativo en sujetos enfermos de SCA2 ha demostrado una disminución significativa en la actividad funcional de las enzimas 
glutation S-transferasa (GST), superóxido-dismutasa (SOD) y catalasa (CAT), lo que explica la presencia de estrés oxidativo de clasificación crítica en los pacientes, expresado por el incremento significativo de marcadores de daño oxidativo a proteínas, lípidos y al ADN. ${ }^{20,21}$

De igual manera se ha observado una disminución significativa de los niveles de $\mathrm{Zn}$, $\mathrm{Cu}$ y $\mathrm{Fe}$ en el suero y el líquido cefalorraquídeo de los pacientes, ${ }^{22}$ lo que pudiese guardar relación con la alteración genética que provoca la enfermedad. Es probable que la ataxina- 2 mutada y agregada en forma de inclusiones pudiera atrapar estos metales divalentes formando complejos de poca solubilidad y limitando su utilización en los procesos fisiológicos donde estos se requieren. Este resultado genera el primer ensayo clínico controlado en pacientes cubanos mediante la suplementación de sulfato de cinc.

\section{Caracterización neurofisiológica de la SCA2}

Entre los aportes más significativos que el CIRAH ha brindado al conocimiento sobre la SCA2 y su aplicación en estrategias terapéuticas resalta la identificación de variables electrofisiológicas como marcadores objetivos del daño genético y la progresión de la enfermedad, así como la obtención de marcadores preclínicos de elevado valor pronóstico. Estos resultados han sido presentados a la comunidad científica nacional e internacional en más de 50 artículos científicos, muchos de los cuales han sido publicados en revistas internacionales de alto impacto como Annals of Neurology, Movement Disorders y la revista de la Academia de Ciencias de Nueva York.

Los principales resultados en este sentido son la identificación de la neuropatía periférica axonal sensitiva como biomarcador preclínico y de progresión de la enfermedad como resultado de una investigación longitudinal de más de 20 años. ${ }^{23,24}$ Esta alteración neurofisiológica es susceptible a tratamiento farmacológico, por lo que también puede clasificarse como un importante blanco terapéutico (Fig.3).

A

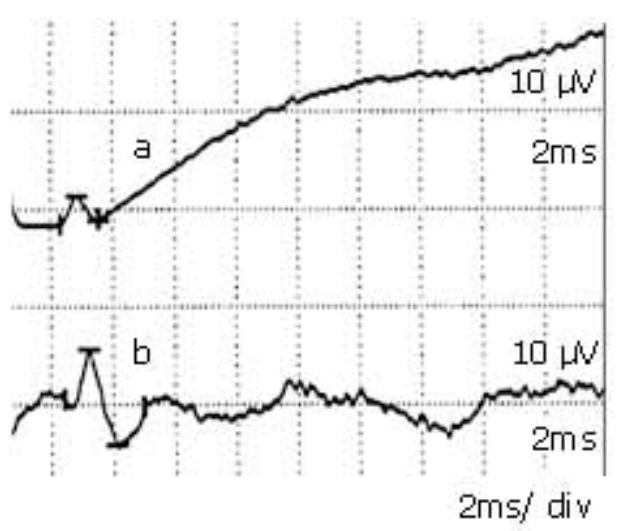

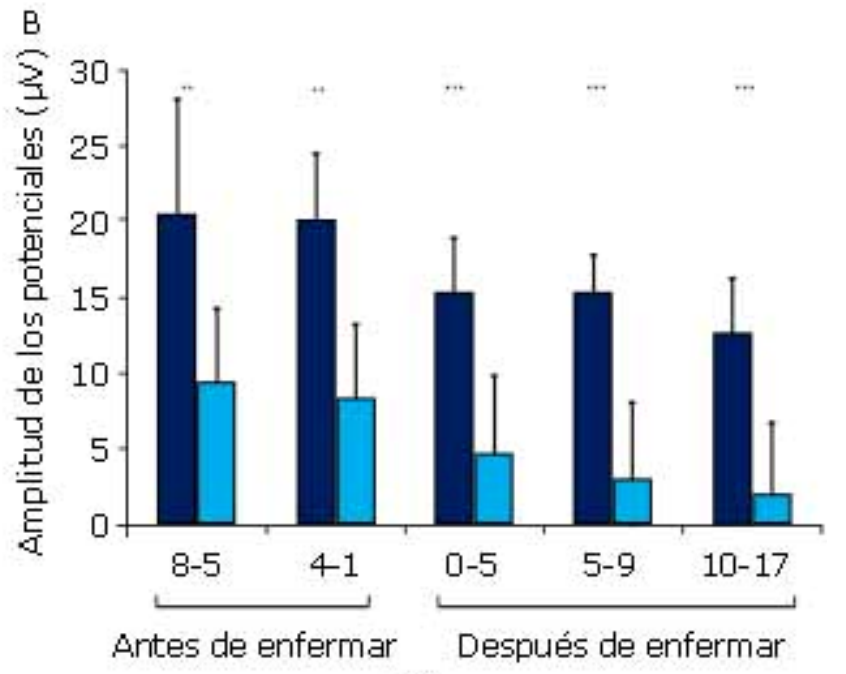

Años

Fig. 3. Neuropatía sensitiva axonal en la SCA2. A) Disminución de la amplitud del potencial sensitivo del nervio mediano en un paciente con SCA.2 ( 35 años de edad, tiempo de evolución 5 años y 40 de repeticiones de $\mathrm{CAG}$ ) comparado con un control sano de la misma edad. B) Progresión de neuropatía sensitiva axonal de nervio mediano durante 20 años de evaluación electrofisiológica. 
Otros resultados relevantes del CIRAH es la identificación de la velocidad de los movimientos oculares sacádicos como la mejor variable endofenotípica de la SCA2, pues guarda una estrecha relación con el tamaño de la expansión poliglutamínica siendo un biomarcador de daño genético más objetivo que la edad de inicio. ${ }^{25}$ Estudios más recientes describieron la utilidad de esta variable como marcador preclínico de la SCA2, lo que le confiere una importancia adicional en el pronóstico y diagnostico de la enfermedad. ${ }^{26}$ De igual forma se ha identificado una prolongación significativa de la latencia de los movimientos oculares sacádicos (Fig.4), la que correlaciona con el grado de disfunciones frontoejecutivas en los pacientes con SCA2. ${ }^{27}$
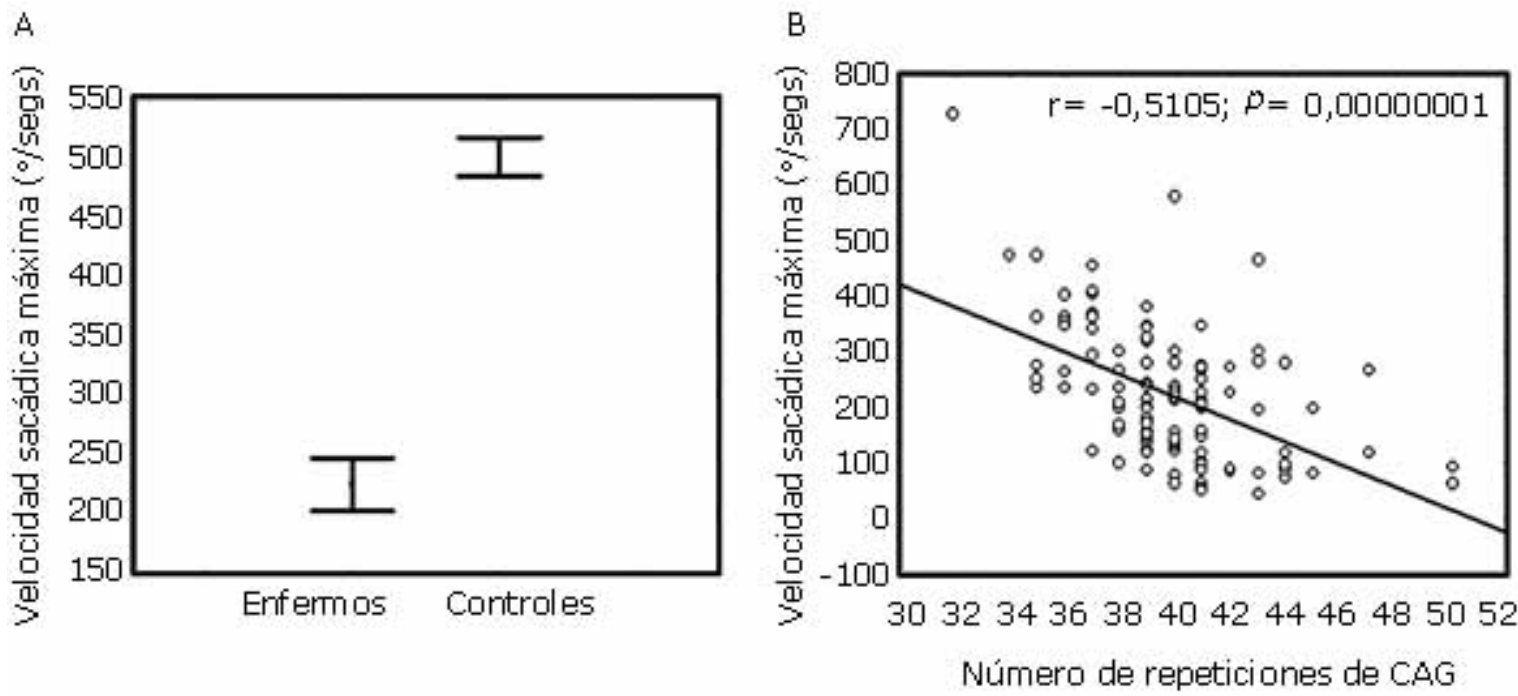

Fig. 4. La velocidad sacádica como marcador del daño genético en la SCA2. A) Disminución estadísticamente significativa de la velocidad sacádica a $60 \% / 5$ en los pacientes con respecto a los sujetos controles. B) Correlación negativa significativa entre la velocidad sacádica a $60 \%$ y el número de repeticiones del CAG en los enfermos.

El estudio y caracterización de trastornos de sueño en pacientes cubanos con SCA2 y sujetos presintomáticos ha sido otra de las principales contribuciones del grupo cubano. Los estudios realizados en otros países incluyen pocos pacientes y en todos los casos sugieren la confirmación de los resultados en la cohorte cubana. El estudio de 32 enfermos cubanos y 36 presintomáticos permite caracterizar con un mayor grado de profundidad los trastornos del sueño REM, identificándola como un buen marcador preclínico y de progresión de la enfermedad. Adicionalmente, de manera distintiva se identifica la existencia de movimientos periódicos de las extremidades en el $38 \%$ de los enfermos, lo que resulta en un nuevo blanco terapéutico, teniendo en cuenta que este trastorno motor del sueño puede ser tratado con agonistas dopaminérgicos. ${ }^{28,29}$

\section{Alternativas terapéuticas para la SCA2}

Hasta este momento, no se cuenta con un tratamiento efectivo que modifique el curso progresivo de la SCA2, aunque se están realizando numerosos esfuerzos encaminados a la identificación de blancos terapéuticos y las consiguientes estrategias de intervención. 
A pesar de lo anterior, se ha informado que mediante un tratamiento dopaminérgico y anticolinérgico se logra reducir el temblor, la distonía y la bradicinecia en pacientes con SCA $2,{ }^{30}$ mientras que las contracciones musculares dolorosas se pueden aliviar con magnesio, quinina o mexiletina. ${ }^{31}$ Las manifestaciones parkisonianas mejoran con la administración de levodopa o agonistas de la dopamina. ${ }^{32}$ Lamentablemente, estas manifestaciones clínicas no son de las más comunes en los pacientes con SCA2.

Indudablemente, Cuba cuenta con las mejores condiciones para ejecutar ensayos clínicos terapéuticos contra la SCA2. Aquí se concentra la mayor población de enfermos, la que ha sido muy bien caracterizada desde un punto de vista epidemiológico, clínico, electrofisiológico, bioquímico y molecular. Además, el CIRAH cuenta con un sistema de salud e investigación científica que le permiten llevar a cabo de manera integrada todas las acciones necesarias para tales proyectos.

En estos momentos, el Centro, en estrecha asociación con varias instituciones de la isla, tales como el CIDEM y el Centro Coordinador de Ensayos Clínicos, está inmerso en la realización de diversos ensayos clínicos controlados como resultado fehaciente de los esfuerzos que se realizan desde hace más de 3 décadas para darle solución al serio problema de salud que representa esta enfermedad.

\section{La rehabilitación como estrategia terapéutica para la SCA2}

Es conocido que el ejercicio terapéutico constituye uno de los pilares fundamentales en el tratamiento físico de los pacientes con afecciones neurodegenerativas, afirmación que ha sido recientemente apoyada por el concepto de neuroplasticidad. ${ }^{33}$

A pesar de ello, prácticamente no existen publicaciones que asocien a la rehabilitación física como una estrategia terapéutica para la SCA2. Una vez más, Cuba se convierte en vivo ejemplo a nivel internacional en cuanto a su contribución asistencial y social a las familias afectadas.

Desde el año 1998 en Cuba se aplica un programa multifactorial para la rehabilitación de los individuos afectados por ataxias hereditarias. ${ }^{34}$ El mismo consta de múltiples sesiones de rehabilitación física, defectológica, logopédica y psicológica. Actualmente más de 400 pacientes han recibido los beneficios de este programa.

Además del programa de rehabilitación integral para las ataxias hereditarias, Cuba cuenta con un sistema objetivo para su evaluación desde el punto de vista clínico, cuantitativo, bioquímico y electrofisiológico. Los resultados obtenidos con este sistema indican que el $68 \%$ de los pacientes rehabilitados mejoran algún parámetro clínico. ${ }^{35}$ Esta mejoría contribuye a la elevación de la calidad de vida de los individuos enfermos al reducir la invalidez propia de la enfermedad y la dependencia que de ella se deriva. Sin embargo, es propicio aclarar que para obtener estos beneficios, es necesaria la correcta dosificación y sostenibilidad de las sesiones de rehabilitación. Por esta razón, actualmente se están desarrollando las acciones necesarias para extender el programa de rehabilitación multifactorial para la SCA2 a todas las comunidades del país.

\section{Diagnóstico prenatal y presintomático de la SCA2}

La incertidumbre que ronda a los individuos con riesgo de padecer alguna enfermedad hereditaria ha exigido de la comunidad científica internacional un esfuerzo por implementar múltiples sistemas de diagnóstico molecular para muchas de estas afecciones. 
Cuba cuenta con el único programa de test predictivo para la SCA2 a nivel internacional. El mismo posibilita el diagnóstico prenatal y presintomático de esta enfermedad, aparejado con el consiguiente asesoramiento genético, seguimiento psicológico y apego a las normas éticas y confidenciales. ${ }^{36,37}$

Durante los 10 años de creado este programa, las demandas de participación han sido elevadas debido a la transmisión de las experiencias de los participantes, las campañas educacionales realizadas, la larga espera por la disponibilidad del diagnóstico, el buen nivel socioeducacional de la población, las sesiones de asesoramiento genético y la salud gratuita al alcance de todos.

En un estudio preliminar realizado sobre el impacto de realización del test predictivo se describe una disminución de los niveles de ansiedad y depresión durante el curso del programa. Una vez realizado el estudio casi todos los participantes experimentan impacto psicológico favorable con el diagnóstico. No se registran eventos catastróficos ni conductas suicidas, o episodios psiquiátricos severos que requirieran hospitalización. ${ }^{36,37}$

\section{Atención social a las familias afectadas}

Los estudios neuroepidemiológicos realizados en Holguín no solo se concentraron en la caracterización clínica, genealógica y molecular de los sujetos enfermos, sino que profundizan en variables sociodemográficas como el grado de escolaridad, la profesión y las condiciones de vivienda, lo que sienta las bases necesarias para el desarrollo de un programa de atención social a las familias más necesitadas, que incluye la ubicación de cuidadoras, la entrega y reparación de viviendas, las prestaciones monetarias, entre otras. Con la instauración del Programa de los Trabajadores Sociales se logra una atención social más especializada que beneficia a un número mayor de familias con la entrega de equipos electrodomésticos, andadores, camas y dietas especiales.

Por otro lado, el CIRAH ha organizado y fomentado el desarrollo de otras actividades sociales como cumpleaños colectivos, bodas, actividades deportivas y culturales y la creación de grupos de autoayuda.

\section{Perspectivas de trabajo del CI RAH}

Las perspectivas de trabajo del CIRAH se encaminan a perfeccionar los programas de neurorehabilitation y de diagnóstico prenatal y presintomático, la identificación de nuevos blancos terapéuticos y biomarcadores de la enfermedad a partir de la instauración de nuevas técnicas para la investigación y el diagnostico, así como el desarrollo de nuevos ensayos clínicos controlados y la continuidad de las definiciones de los principales mecanismos patogénicos de esta enfermedad. Además, se pretende extender las acciones investigativas asistenciales a otras neurodegeneraciones de alta prevalencia en Holguín, como las enfermedades de Parkinson y Huntington.

Para ello, la institución ha proyectado priorizar el establecimiento de proyectos de colaboración con otros centros del país y con instituciones extranjeras a través de redes multicentros para las investigaciones y tratamiento de las ataxias hereditarias.

Sin lugar a dudas, los resultados obtenidos de las investigaciones fenotípicas y genotípicas, así como las intervenciones médicas asistenciales, junto al programa social de atención directa a las familias afectadas y la integración de la dirección de salud, del gobierno y el Partido, le confieren al CIRAH una posición única a nivel internacional, que le hacen Centro de Referencia Nacional e Internacional para el 
abordaje de las ataxias hereditarias y un modelo para el estudio de las enfermedades neurodegenerativas.

\section{REFERENCI AS BI BLI OGRÁFICAS}

1. Velázquez L. Ataxia Espinocerebelosa tipo 2. Principales aspectos neurofisiológicos en el diagnóstico, pronóstico y evolución de la enfermedad. Holguín: Ediciones Holguín; 2008.

2. Palau F, Espinos C. Autosomal recessive cerebellar ataxias. Orphanet J Rare Dis. 2006; 17: 1-47

3. Matilla Dueñas A, Sánchez I, Corral Juan M, Dávalos A, Álvarez R, Latorre P. Cellular and molecular pathways triggering neurodegeneration in the spinocerebellar ataxias. Cerebellum. 2010;9(2): 148-66.

4. Durr A. Autosomal dominant cerebellar ataxias: polyglutamine expansions and beyond. Lancet Neurol. 2010; 9:88594.

5. Vallés L, Estrada GL, Bastecherrea SL. Algunas formas de heredoataxia en una región de Cuba. Rev Cubana Neurol. 1978; 27:163-76.

6. Orozco DG, Nodarse FA, Cordovés R, Aurburger G. Autosomal dominant cerebellar ataxia: Clinical analysis of 263 patients from a homogeneous population in Holguin, Cuba. Neurology. 1990; 40(90): 1369-75.

7.VelázquezPérez L, Santos FN, García R, Paneque HM, Hechavarría PR. Epidemiología de la Ataxia Cubana. Rev Neurol. 2001; 32 (7): 606-11.

8. Velázquez Pérez L, Cruz GS, Santos Falcón N, Almaguer LE, Escalona K, Rodríguez R. Molecular epidemiology of spinocerebellar ataxias in Cuba: Insights into SCA2 founder effect in Holguin. Neurosci Lett. 2009a; 454(2): 157-60.

9. Sánchez Cruz G, Velázquez Pérez L, Gómez Peña L, Martínez Góngora E, Castellano Sánchez G, Santos Falcón N. Manifestaciones disautonómicas en pacientes con ataxia espinocerebelosa tipo 2 cubana. Rev Neurol. 2001;33(5):428-33.

10. Julio MB, Gilberto SC, Andres MG, Mario EB, Luis VP. Heart rate variability in type 2 spinocerebellar ataxia. Acta Neurol Scand. 2010. doi: 10.1111 D j.16000404.2009.01320.

11. Burk K, Globas C, Bosch S, Graber S, Abele M, Brice A, et al. Cognitive deficits in spinocerebellar ataxia type 1, 2, and 3. J Neurol. 2003;250:207-11.

12. Gispert S, Twells R, Orozco G, Brice A, Weber J, Heredero L, et al. Chromosomal assignment of the second locus for autosomal dominant cerebellar ataxia (SCA2) to chromosome 12q23-24.1. Nat Genet. 1993;4(3):295-9.

13. Hernandez A, Magarino C, Gispert S, Santos N, Lunkes A, Orozco G, et al. Genetic mapping of the spinocerebellar ataxia 2 (SCA2) locus on chromosome 12q23-q24.1. Genomics. 1995; 25(2):433-5.

http://scielo.sld.cu 


\section{Revista Cubana de Salud Pública. 2011;37(3):230-244}

14. Pulst MS, Nechiporuk A, Nechiporuk T. Moderate expansion of a normally biallelic trinucleotide repeat in spinocerebellar ataxia type 2 . Nature Genet 1996; 14: 269276.

15. Huynh DP, Del Bigio MR, Ho DH, Pulst SM. Expression of ataxin-2 in brains from normal individuals and patients with Alzheimer's disease and spinocerebellar ataxia 2. Ann Neurol. 1999; 45:232-41.

16. Sahba S, Nechiporuk A, Figueroa KP, Nechiporuk T, Pulst SM. Genomic structure of the human gene for spinocerebellar ataxia type 2 (SCA2) on chromosome 12q24.1. Genomics. 1998;47: 359-64.

17. Pulst SM, Santos N, Wang D, Yang H, Huynh D. Spinocerebellar Ataxia type 2: PolyQ Repeat Variation in the CACNA1A Channel Modifies Age of Onset. Brain. 2005; 128: 2297-303.

18. Simon DK, Zheng K, Velazquez L, Figueroa KP, Falcón N, Almaguer LE, et al. Mithochondrial complex I gene variant associated with early age of onset in SCA2. Arch Neurol. 2007; 64: 10424.

19. Aguiar J, Fernandez J, Aguilar A. Ubiquitous expression of human SCA2 gene under the regulation of the SCA2 self promoter cause specific Purkinje cell degeneration in transgenic mice. Neuroscien Let. 2006;392:202-6.

20. Almaguer L, Almaguer D, González Y, Martínez E, Valcárcel P. Capacidad antioxidante total en pacientes cubanos con ataxia Espinocerebelosa tipo 2. Rev Mex Neuroci. 2005; 6(3): 201-6.

21. Almaguer Mederos LE, Rodríguez Almira Y, Martínez Góngora E. Estudio preliminar de la prevalencia de anticuerpos antigliadina en pacientes cubanos con ataxia espinocerebelosa tipo 2. Rev Mex Neurocien. 2006; 7(1):76-9.

22. González C, Sánchez G, González Quevedo A, Delgado R, Velázquez L, García Rodríguez JC, et al. Serum and cerebrospinal fluid levels of copper, iron and zinc in patiens with Ataxia type SCA-2 from the province of Holguin in Cuba. Therapeutic Basic Dialogues Clin Neuroscien. 2005; 13(4): 12-6.

23. Velázquez Pérez L, Sánchez Cruz G, Canales Ochoa N, Rodríguez Labrada R, Rodríguez Díaz J, Almaguer Mederos L, et al. Electrophysiological features in patients and presymptomatic $r$ relatives with Spinocerebellar Ataxia type 2. J Neurol Sci. 2007; 263: 158-64.

24. Velazquez Pérez L, Rodriguez Labrada R, Canales Ochoa N, Sanchez Cruz G, Fernandez Ruiz J, Montero JM, et al. Progression markers of Spinocerebellar ataxia 2. A twenty years neurophysiological follow up study. J Neurol Sci. 2010;290:22-6.

25. Velazquez Pérez L, Seifried C, Santos Falcón N, Abele M, Ziemann U, Almaguer $\mathrm{LE}$, et al. Saccadic velocity is controlled by polyglutamine size in spinocerebellar ataxia 2. Ann Neurol. 2004;56(3):444-7.

26. Velázquez Pérez L, Seifried C, Abele M, Wirjatijasa F, Rodríguez Labrada R, Santos Falcón $\mathrm{N}$, et al. Saccadic velocity is reduced in presymptomatic spinocerebellar ataxia type 2. Clin Neurophysiol. 2009; 120(3):632-5. 


\section{Revista Cubana de Salud Pública. 2011;37(3):230-244}

27. Rodríguez Labrada R, Velázquez Pérez L, Seigfried C, Canales Ochoa N, Auburger G, Medrano Montero J, et al. Saccadic latency is prolonged in Spinocerebellar Ataxia type 2 and correlates with the frontal-executive dysfunctions. J Neurol Sci. 2011. doi: 10.1016/j.jns.2011.03.033.

28. Velázquez Pérez L, Voss U, Rodríguez Labrada R, Auburger G, Canales Ochoa N, Sánchez Cruz G, et al. Sleep disorders in Spinocerebellar Ataxia Type 2 Patients. Neurodeg Dis. 2011 doi: 10.1159/000324374.

29. Rodriguez Labrada R, Velazquez Pérez L, Canales Ochoa N, Galicia Polo L, Haro Valencia R, Sánchez Cruz G, et al. Subtle REM Sleep Abnormalities in Presymptomatic Spinocerebellar Ataxia Type 2 Gene Carriers. Mov Disord. 2011;26:347-50.

30. Nandagopal R, Moorthy SG. Dramatic levodopa responsiveness of dystonia in a sporadic case of spinocerebellar ataxia type 3. Postgrad Med J. 2004;80:3635.

31. Furtado S, Farrer M, Tsuboi Y. SCA-2 presenting as parkinsonism in an Alberta family: clinical, genetic and PET findings. Neurology. 2002; 59: 16257.

32. Kanai K, Kuwabara S, Arai K, Sung JY, Ogawara K, Hattori T. Muscle cramp in Machado-Joseph disease: altered motor axonal excitability properties and mexiletine treatment. Brain. 2003; 126: 96573.

33. Kottke FJ, Stillwell GK, Lehmann Krusen JF. El ejercicio terapéutico para mantener la movilidad. En: Krusen, editor Medicina Física y Rehabilitación. Buenos Aires: Editora Médica Panamericana; 1990. p. 431

34. Pérez Ávila I, Fernández Vieitez J A, MartínezGóngora E, Ochoa Mastrapa R, Velázquez Manresa MG. Efectos de un programa de ejercicios físicos sobre variables neurológicas cuantitativas en pacientes con ataxia espinocerebelosa tipo 2 en estadio leve. Rev Neurol. 2004;39:907-10.

35. Rodríguez Díaz JC, Velázquez Pérez L, Sánchez Cruz G, Almaguer Gotay D, Rodríguez Labrada R, Aguilera Rodríguez R, et al. Evaluation of Neurological Restoration in patients with Spinocerebellar Ataxia type 2. Plast Rest Neurol. 2008; 7: 13-8.

36. Paneque HM, Prieto AL, Reynaldo RR. Psychological aspects of presymptomatic diagnosis of Spinocerebellar Ataxia Type 2 in Cuba. Commun Genet. 2007; 10: 1329.

37. Milena Paneque M, Lemos C, Sousa A, Velazquez I, Fleming M, Sequeiros J. Role of the Disease in the Psychological Impact of Pre-Symptomatic Testing for SCA2 and FAP ATTRV30M: Experience with the Disease, Kinship and Gender of the Transmitting Parent. J Genet Counsel. 2009; 18: 48393.

Recibido: 28 de Octubre de 2010.

Aprobado 9 de noviembre de 2010. 
Luis Velázquez Pérez. Avenida Los Álamos No. 25 e/ Independencia y Constitución. Holguín 80100, Cuba.

Teléf.: 24-462296 y 24 424090. Correo electrónico: cirahsca2@cristal.hlg.sld.cu 